\title{
Structure elucidation of female-specific volatiles released by the parasitoid wasp Trichogramma turkestanica (Hymenoptera: Trichogrammatidae)
}

\author{
Armin Tröger ${ }^{1}$, Teris A. van Beek ${ }^{2}$, Martinus E. Huigens ${ }^{3}$, \\ Isabel M. M. S. Silva ${ }^{3}$, Maarten A. Posthumus ${ }^{2}$ and Wittko Francke ${ }^{* 1}$
}

\section{Full Research Paper}

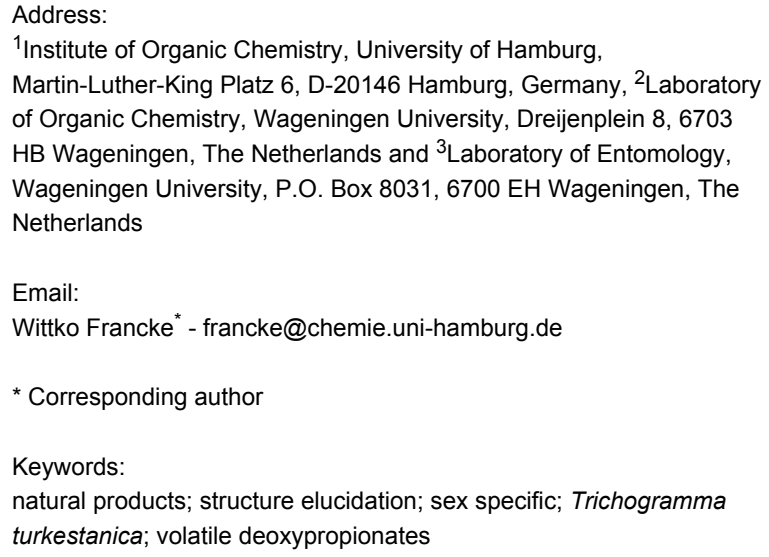

\begin{abstract}
Females of the parasitoid wasp Trichogramma turkestanica produce the putative polydeoxypropionates $(2 E, 4 E, 6 S, 8 S, 10 S)$ 4,6,8,10-tetramethyltrideca-2,4-diene and $(2 E, 4 E, 6 S, 8 S, 10 S)$-4,6,8,10-tetramethyltrideca-2,4-dien-1-ol or their enantiomers as sex specific volatiles. The structures were assigned on the basis of GC-MS investigations using synthetic reference compounds.
\end{abstract}

\section{Introduction}

Wasps of the genus Trichogramma (Hymenoptera: Trichogrammatidae) are egg parasitoids of insect eggs [1]. They are commercially used as biological control agents against many insect pests, worldwide [2]. The taxonomy of Trichogramma wasps is hampered by their particularly small size and by strong morphological similarities between the species. Several attempts, including chemotaxonomic approaches, have been made to solve this problem [3-5]. While it is generally accepted that parasitoid wasps use chemical cues for host location [6], little is known about (volatile) semiochemicals playing a role in intraspecific communication [7-11].

Based on bioassays with Trichogramma turkestanica and extensive analytical investigations, two female-specific volatile compounds have been described as possible pheromone components and were tentatively identified as the hydrocarbon 2,6,8,12-tetramethyltrideca-2,4-diene (A) and the corresponding allylic alcohol 2,6,8,12-tetramethyltrideca-2,4-diene-1- 
ol (B) [12]. Since the two proposed structures were deduced from analytical data only, the aim of the present study was to scrutinize these suggestions by unambiguous synthesis.

\section{Results and Discussion}

The $70 \mathrm{eV}$ EI mass spectra of the two natural products $\mathbf{A}$ and $\mathbf{B}$ are depicted in Figure 1 [12]. Because the carbon skeleton had been reported to be the same in both compounds, we used the hydrocarbon $\mathbf{A}$ as the first target for the preparation of a reference sample. As an initial approach we aimed at the synthesis of a library of stereoisomers of A (Figure 2).

The commercially available racemic anti-dimethyl 2,4dimethylglutarate (1) was reduced to the corresponding racemate of 2,4-dimethylpentan-1,5-diol (2) [13], which was further transformed to the monosilyl ether $\mathbf{3}$ and subsequently to the corresponding tosylate 4. Using 3-methylbutylmagnesium bromide and applying cuprate chemistry [14], 4 was chain elongated to 5, which was deprotected to yield anti-2,4,8-trimethylnonanol (6). After oxidation to the corresponding aldehyde, the stereogenic center at position 2 underwent ca. $6 \%$ epimerization to yield 7 as a mixture of 4 stereoisomers. The synthesis of 2,6,8,12-tetramethyltrideca-2,4-diene (8) was completed by Wittig reaction using (3-methylbut-2-en-1-yl)triphenylphosphonium bromide [15]. As expected, the obtained mixture of all possible stereoisomers of $\mathbf{8}$ showed an E/Z-ratio of ca. 2:1 and was highly dominated by the anti-isomers (see Supporting Information File 1, Figure S1). The E/Z-isomers of the two pairs of diastereomers could be separated by gas chromatography. Analysis by coupled gas chromatography/mass spectrometry (GC-MS) showed the $70 \mathrm{eV}$ EI mass spectra of the synthetic compounds to be almost identical. Though the mass spectra of 8 (Figure 3) and that of the natural product A (Figure 1) showed strong similarities, small but decisive qualitative and quantitative differences in the fragmentation pattern were obvious. In contrast to the mass spectrum of $\mathbf{A}$, those of $\mathbf{8}$ showed an additional signal at $m / z 180$ (loss of isobutene), whereas the signal at $m / z$ 153, present in the spectrum of $\mathbf{A}$ was absent. In addition, the pair at $\mathrm{m} / z 67$ and $\mathrm{m} / \mathrm{z} 69$ showed different relative abundances.
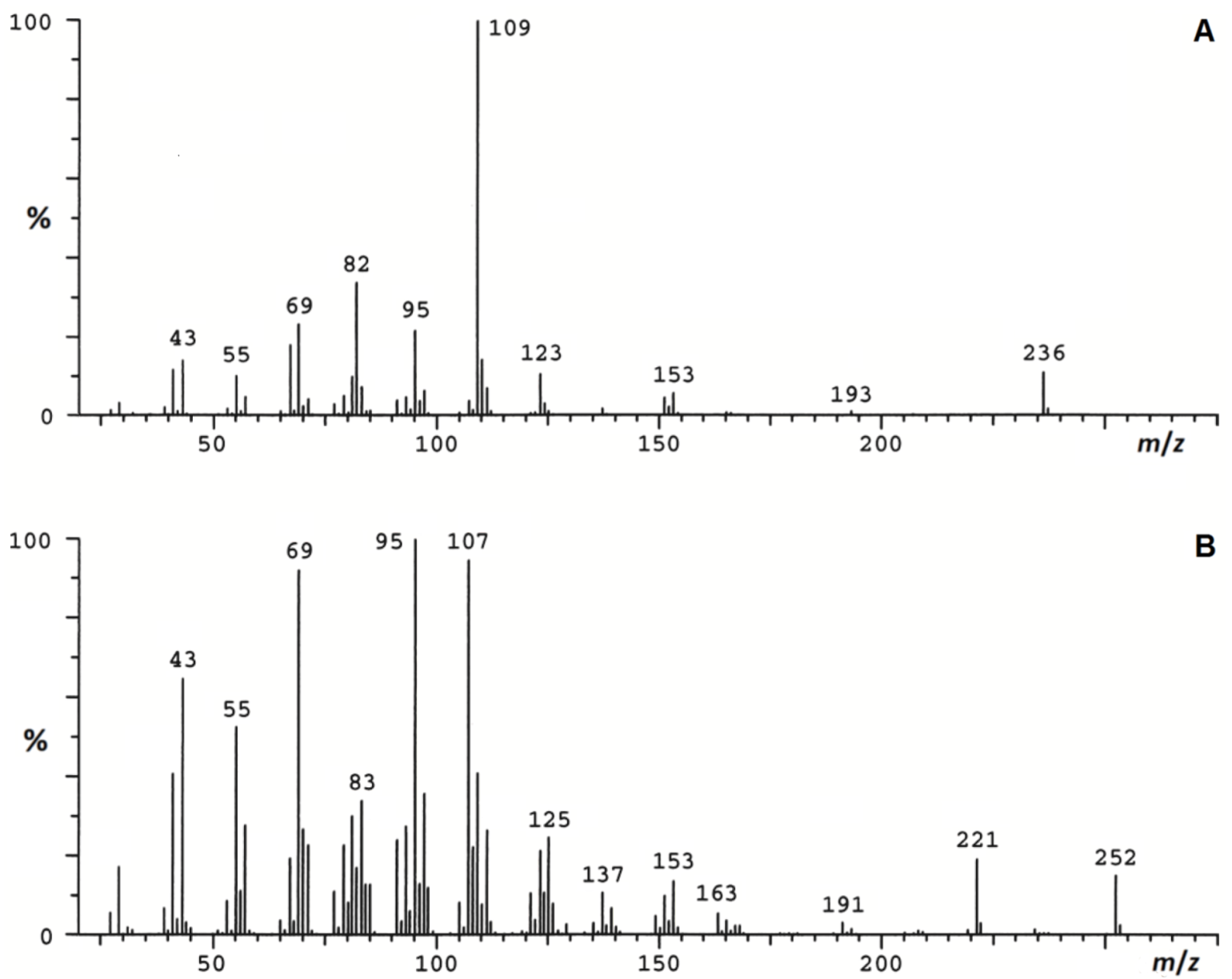

Figure 1: Published 70 eV El mass spectra of the naturally occurring compounds $\mathbf{A}$ and $\mathbf{B}$ [12]. 


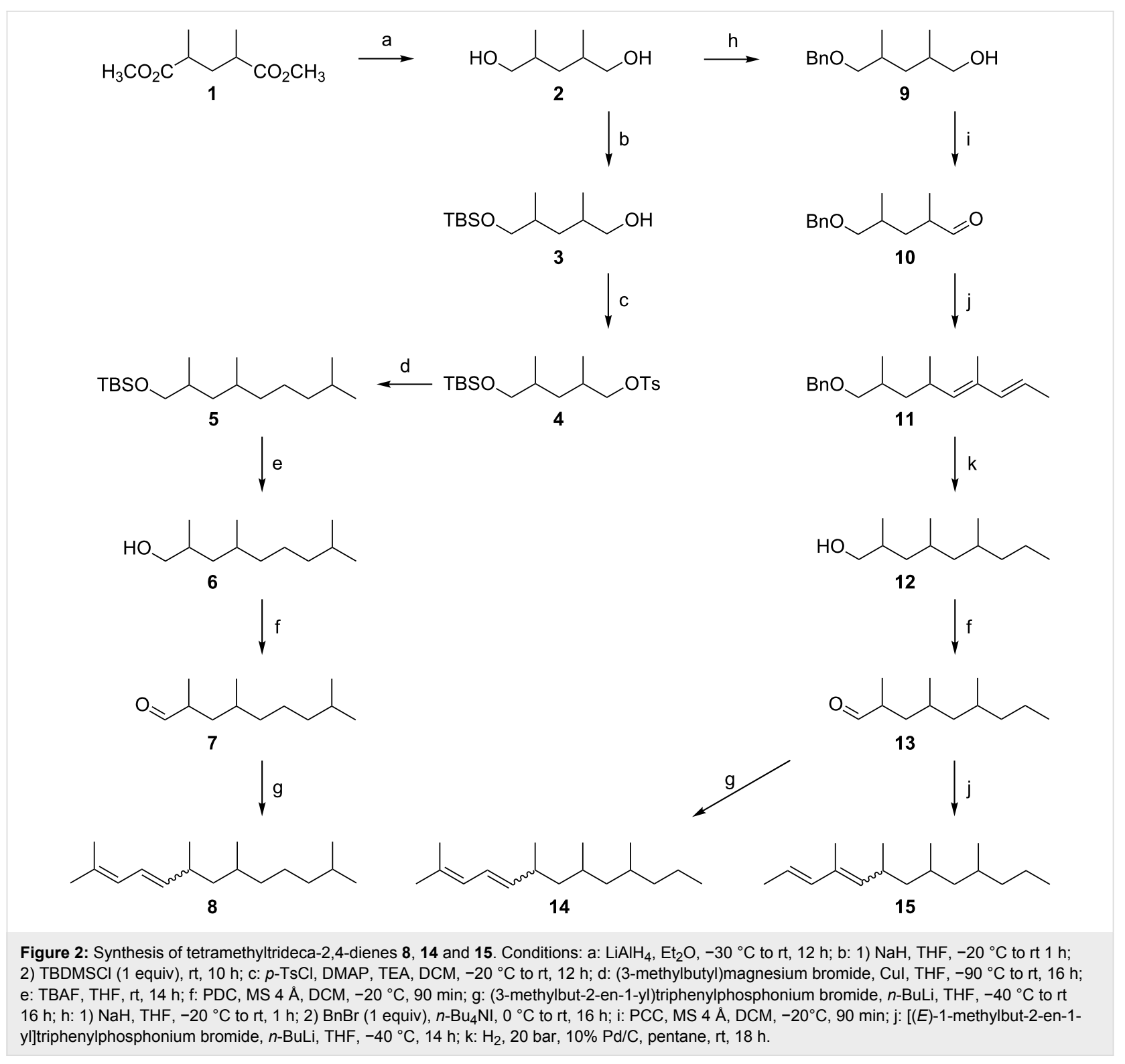

Thus, it was obvious that the structures published for the natural products needed modification. Sticking to the basic carbon skeleton proposed for A and B [12], we looked for a plausible biogenetic way providing an alternative structure for $\mathbf{A}$ from reasonable building blocks without too much changing of $\mathbf{8}$. Methyl branching at positions 6 and 8 was indicated by pronounced signals at $\mathrm{m} / \mathrm{z} 123=\mathrm{M}^{+}-113$ and $\mathrm{m} / \mathrm{z} 151=$ $\mathbf{M}^{+}-85$ in the mass spectrum of $\mathbf{A}$ [12] (Figure 1), which we regarded to result from loss of the last eight or six carbons, respectively. Therefore, we kept this feature in the structure of the next candidate. Leaving the dienic system unchanged, we consequently decided to shift the methyl group from position 12 in 8 to position 10. As a result, our next candidate was $2,6,8,10-$ tetramethyltrideca-2,4-diene (14), because the formation of such a polydeoxypropionate by condensation of four units of methyl- malonate, followed by chain elongation with malonate and another methylmalonate (including deoxygenation steps) seemed to be plausible [16]. These biogenetic considerations excluded methyl groups at positions 9 and 11 .

To prepare a mixture of all stereoisomers of 14, we again used 2 as the starting material (Figure 2). Monobenzylation to $\mathbf{9}$, followed by oxidation, yielded the aldehyde 10, which was chain elongated by Wittig reaction with [(2E)-1-methylbut-2-en-1yl]triphenylphosphonium bromide [17] to afford the protected dienol 11 as a mixture of 4 racemates. This was due to partial epimerization at position 2 of the aldehyde $\mathbf{1 0}$ and the less selective Wittig coupling. Consequently, hydrogenation of $\mathbf{1 1}$ yielded a mixture of all 8 possible stereoisomers of the saturated alcohol 12; for relative proportions, see Supporting Infor- 


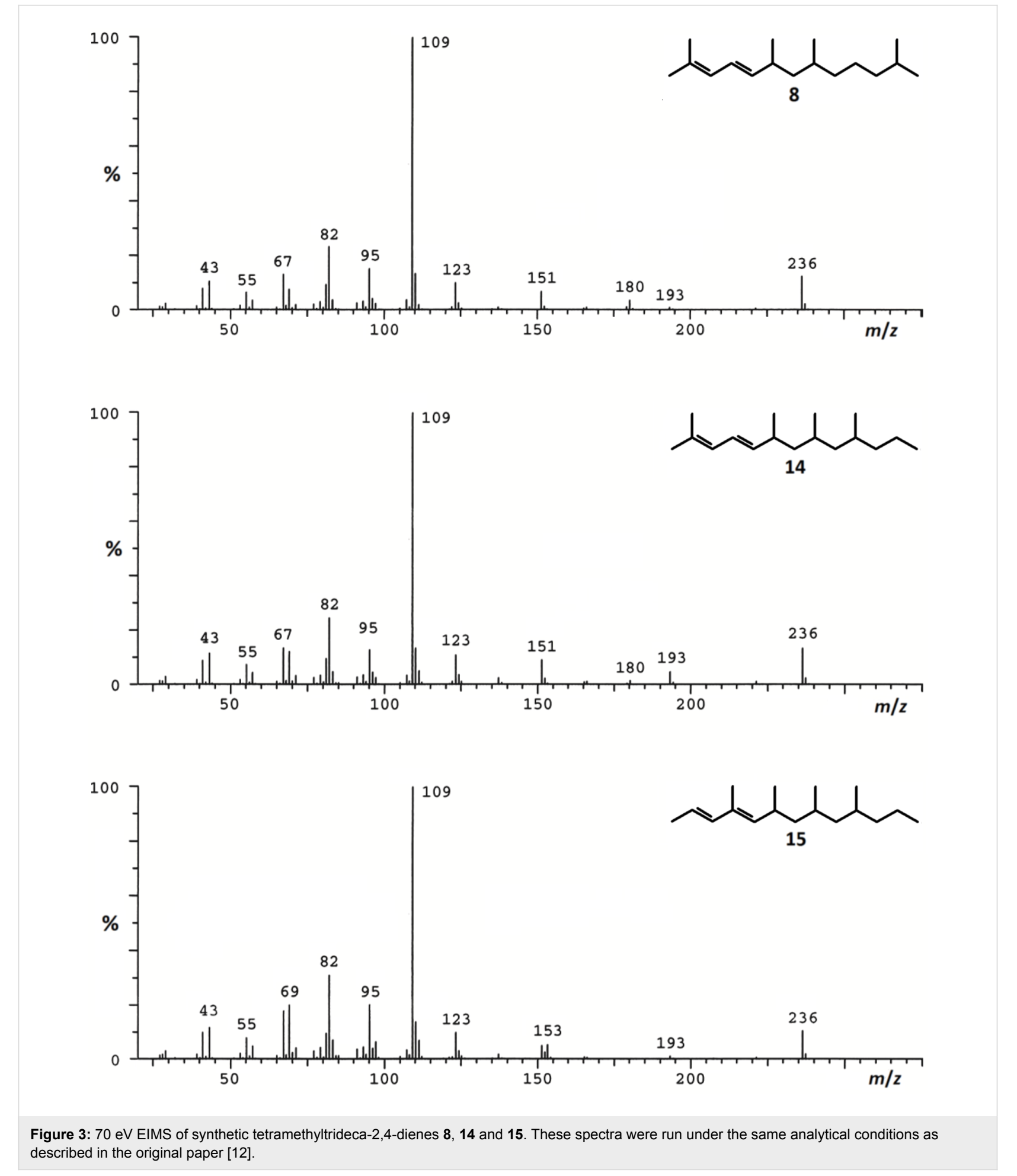

mation File 1. Starting from $\mathbf{1 2}$ and following the sequence described for the transformation of $\mathbf{6}$ to $\mathbf{8}$, a mixture of the stereoisomers of the target diene $\mathbf{1 4}$ was obtained via 2,4,6trimethylnonanal (13) [15]. GC-MS-analysis of this mixture revealed a 3:1 dominance of the $E$-configurated racemates over the earlier eluting $Z$-series. The chromatogram showed seven well-separated peaks, indicating that only two isomers co-eluted (see Supporting Information File 1, Figure S1). However, it soon became clear that the natural product was not among the synthetic compounds 14, because their mass spectra basically showed the same differences to that of A (presence of $\mathrm{m} / \mathrm{z} 180$, absence of $\mathrm{m} / \mathrm{z} 153$ ) as did the 2,6,8,12-tetramethyltrideca-2,4- 
dienes (8) (Figure 1 and Figure 3). In the next approach we considered a structural change in the dienic system of $\mathbf{1 4}$ as an additional modification of the published structure of $\mathbf{A}$.

As indicated above, the formation of the signal at $m / z 180$ in the mass spectra of $\mathbf{8}$ and $\mathbf{1 4}$ may be due to the loss of isobutene which would be avoided by a shift of the methyl group from carbon 2 to positions 4 or 5 . Results of derivatization reactions of the natural product by Diels-Alder reaction with 4-methyl1,2,4-triazolin-3,5-dione left room for the positioning of a methyl group at carbons 2, 3, 4, or 5 of the chain [12]. Location at position 2 would provide 14, which was already ruled out. According to a conceivable biosynthesis of the natural product from methylmalonate and acetate, positions 3 and 5 would be less favourable [16]. However, linear condensation of five methylmalonate units and a final malonate (and again corresponding deoxygenation steps) would produce the carbon skeleton of a 4,6,8,10-tetramethyltrideca-2,4-diene (15). Thus, from a biosynthetic point of view, the structure would be reasonable and follow established principles.

To test the validity of our assumption, we transformed $\mathbf{1 3}$ into 15 by Wittig reaction using [(E)-1-methylbut-2-en-1-yl]triphenylphosphonium bromide [17] in the chain elongation step (Figure 2). The reaction yielded a mixture of products representing two quantitatively different series of eight racemic stereoisomers of 15. According to the $E$-configuration of the double bond in the starting phosphorane and the known $E$-selectivity of the reaction [17], we assigned the $(2 E, 4 E)$-configuration to the more abundant and later eluting series of stereoisomers and the $(2 E, 4 Z)$-configuration to the less abundant one. The diastereomers could be well separated by GC, and only two members of the less abundant $4 Z$-series co-eluted (see Supporting Information File 1, Figure S1). The mass spectra of 15 were the same as that of the natural product (Figure 1 and Figure 3). Gas chromatographic properties on a non-polar DB-1 and a polar Stabilwax (for conditions see ref. [12]) of the first eluting racemate of the more abundant $4 E$-series matched those of the natural product, strongly indicating $(2 E, 4 E)$-configuration of the conjugated double system in the target compound (see Supporting Information File 1, Figure S2).

After the gross structure of the natural product had been found to very likely be $(2 E, 4 E)-4,6,8,10$-tetramethyltrideca-2,4-diene (15), we had to assign the relative stereochemistry at the three stereogenic centers. Actually, we had to decide between 4 structures: syn,syn-, syn,anti-, anti,syn-, and anti,anti-configuration of the methyl groups at carbons 6,8 , and 10 . Since semiochemicals representing saturated polydeoxypropionates typically show syn-configuration, suggesting general principles in enzymatic chain elongation $[16,18]$, we hypothesized
$(2 E, 4 E, 6 S, 8 S, 10 S)$-tetramethyltrideca-2,4-diene or its enantiomer to be the natural product.

To establish a syn,syn-configuration of the three chiral centers in our target compound, we followed the way used by Mori and Kuwahara for the synthesis of the mite pheromone lardolure [19], which we had successfully adopted in the synthesis of vittatalactone [20]. Commercially available 2,4,6-trimethylphenol was hydrogenated to form all-cis-2,4,6-trimethylcyclohexanone. Subsequent Baeyer-Villiger oxidation, followed by reduction of the obtained lactone, yielded syn,syn-2,4-dimethylheptan-1,6-diol 16 (Figure 4). Protection of the primary hydroxy group gave $\mathbf{1 7}$ followed by a Mitsunobu sequence involving the secondary hydroxy group, afforded 18. Chain elongation of 18 by applying cuprate chemistry [14] to its mesylate gave 19 which was deprotected to give 20, which, in turn was oxidized to syn,syn-2,4,6-trimethylnonanal (21). As described for the transformation of $\mathbf{1 3}$ into 15 (Figure 2), 21 was chain-elongated to a 1:3-mixture of $(2 E, 4 Z)$-syn,syn- and (2E,4E)-syn,syn-4,6,8,10-tetramethyltrideca-2,4-diene (22). The stereochemical composition of this defined mixture of stereoisomers of 15 (see Supporting Information File 1, Figure S2) was assigned by NMR spectroscopy, matching respective data reported in the literature $[21,22]$.

The mass spectrum of $(2 E, 4 E)$-syn,syn-4,6,8,10-tetramethyltrideca-2,4-diene (Figure 1 and Figure 3 ) and its gas-chromatographic properties perfectly matched the corresponding data of the natural product $\mathbf{A}$ (linear retention index on DB1 = 1511 and on Stabilwax $=1579$ ) [12]. The structure closely resembles those of the oligomethylpolyenes known as pheromones of nitidulid beetles [24]. The 4-methyl-2E,4E-diene motif has been known as a substructure of pheromones of beetles, cockroaches and scale insects [16]. Whether these compounds are insectproduced or products of (endo)symbionts will need further investigations.

In analogy to the structure of $\mathbf{A}$, compound $\mathbf{B}$ was assigned to be $(2 E, 4 E, 6 S, 8 S, 10 S)-4,6,8,10$-tetramethyltrideca-2,4-dien-1-ol (23) or its enantiomer. According to the procedure described by Markiewicz et al. [23], racemic 23 was prepared by vinylogous Horner-Wadsworth-Emmons reaction of the aldehyde 21 with the anion of diethyl [(2E)-3-methoxycarbonyl-1-methyl-2-en-1yl]phosphonate, followed by reduction of the resulting methyl ester (Figure 4). The obtained product proved to be a mixture of 23 and its $(2 E, 4 Z)$-isomer (23a) in a ratio of about 1:1. For an unambiguous gas-chromatographic discrimination of $\mathbf{2 3}$ and 23a we also synthesized a mixture of the two series of stereoisomers by applying "salt-free" conditions. As expected, the blend was strongly biased by the $(Z$ )-series 23a (see Supporting Information File 1, Figure S3A). The mass spec- 
<smiles>CC(O)CC(C)CC(C)CO</smiles>

16

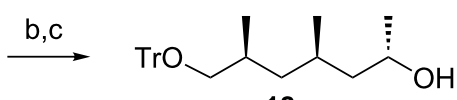

18

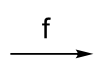<smiles>CCC[C@H](C)CC(C)C[C@H](C)CO</smiles>

20

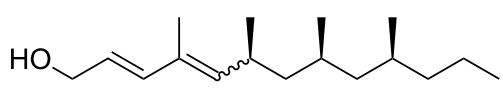

23

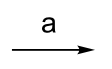<smiles>CC(C[14CH2][Te])C[C@H](C)C[C@@H](C)O</smiles>

17
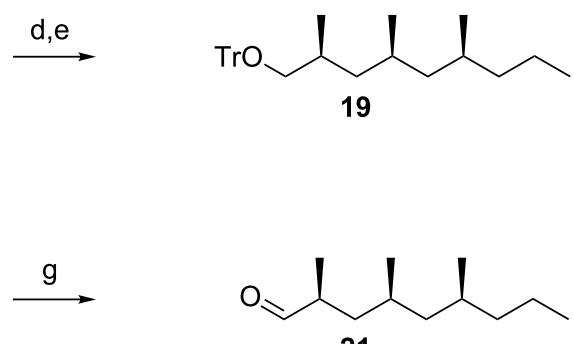

21
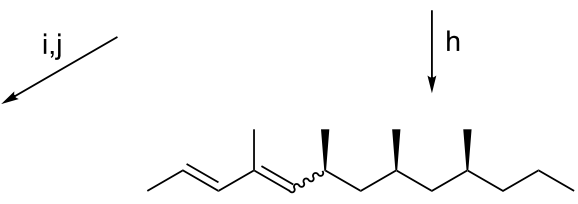

22

Figure 4: Synthesis of (2E,4EZ)-syn,syn-4,6,8,10-tetramethyltrideca-2,4-diene (22), as well as (2E,4E)- and (2E,4Z)- syn,syn-4,6,8,10-tetramethyltrideca-2,4-dien-1-ol (23 and 23a). Conditions: a: TrCl, DMAP, TEA, DCM, rt, 16 h; b: 1) DIAD, TPP, THF, $-20{ }^{\circ} \mathrm{C}, 30 \mathrm{~min}$; 2 ) 3,5-DNBA, rt, 40 min; 3) 17, rt, $17 \mathrm{~h}$; c: $2 \mathrm{~N} \mathrm{NaOH}$ aq, THF/MeOH, rt, $45 \mathrm{~min}$; d: MsCl, TEA, DCM, $-80^{\circ} \mathrm{C}, 2 \mathrm{~h}$; e: $n$-propylmagnesium bromide, Cul, THF, $-90{ }^{\circ} \mathrm{C}$ to rt, $48 \mathrm{~h}$; f: $p$-TsOH, MeOH/THF/H ${ }_{2} \mathrm{O}$, rt, 2 h; g: PDC, MS $4 \AA$, DCM, $-20^{\circ} \mathrm{C}, 90 \mathrm{~min}$; $\mathrm{h}$ : $((E)$-1-methylbut-2-en-1-yl]triphenylphosphonium bromide, $n$-BuLi, LiBr, THF, $-40^{\circ} \mathrm{C}, 12 \mathrm{~h}$; i: 1) diethyl [(1/2EZ)-3-(methoxycarbonyl)-1-methylprop-1/2-en-1-yl]phosphonate (as a mixture of isomers [23]), NaHMDS, LiBr, THF, $\left.-90^{\circ} \mathrm{C}, 1 \mathrm{~h} ; 2\right) 21,-70^{\circ} \mathrm{C}$ to rt, $16 \mathrm{~h}$; j: DIBAIH, DCM, $-80^{\circ} \mathrm{C}$ to rt, $2.5 \mathrm{~h}$.

trum of the $(2 E, 4 E)$-configured 2,4-dien-1-ol 23 (Figure 1), and its gas-chromatographic properties perfectly matched the corresponding data of the natural product $\mathbf{B}$ (linear retention index on DB1 $=1766$ and a Stabilwax $=2317$ ) [12].

Since the preparation of enantiomerically pure syn,syn-2,4,6trimethylnonanal has been described [25-28], our approach includes a formal synthesis of optically active $\mathbf{A}$ and $\mathbf{B}$. Until now, we did not find suitable conditions to separate the enantiomers of the tetramethyldiene $\mathbf{2 2}$ and the respective allyl alcohol 23 (or corresponding derivatives thereof) in order to assign the absolute configuration of the natural products by enantioselective gas chromatography. However, ozonolysis of a natural sample [29] containing the two compounds, followed by reductive work-up, would yield syn,syn-2,4,6-trimethylnonanol, the enantiomers of which can be easily separated by using heptakis-[2,3-di-O-methyl-6-O-(tert-butyldimethylsilyl)]- $\beta$ cyclodextrin $(50 \%$ in OV1701) as the stationary GC phase, operated at $100{ }^{\circ} \mathrm{C}$. Under these conditions, the $(2 S, 4 S, 6 S)$ enantiomer [30] is the later eluting stereoisomer, giving an $\alpha$-value of $\left(t r_{2}: t r_{1}\right)=1.019$ (see Supporting Information File 1, Figure S4).

As a result of our investigations we corrected the structures of the initially published Trichogramma compounds and showed a way to determine the enantiomeric composition of the natural products. Details including the assignment of the absolute configuration of the compounds will be published separately. As yet, the biological significance of the two female-produced compounds is unclear, however, Pompanon et al. [31] showed the presence of a sex specific pheromone released by females of Trichogramma brassicae. Future bioassays with the two new polydeoxypropionates will scrutinize whether they induce courtship behavior in males of Trichogramma turkestanica, as did the extract of females [12].

\section{Supporting Information}

\section{Supporting Information File 1}

Experimental details and characterization data for synthesized compounds.

[http://www.beilstein-journals.org/bjoc/content/ supplementary/1860-5397-10-72-S1.pdf]

\section{References}

1. Flanders, S. E.; Quednau, W. Entomophaga 1960, 5, 285-294. doi:10.1007/BF02372951

2. Smith, S. M. Annu. Rev. Entomol. 1996, 41, 375-406. doi:10.1146/annurev en 41.010196 .002111 
3. Pinto, J. D. Systematics of the North American Species of Trichogramma Westwood (Hymenoptera: Trichogrammatidae; Memoirs of the Entomological Society of Washington, Vol. 22; Entomological Society: Washington, U.S.A, 1999; pp 1-287.

4. Stouthamer, R.; Hu, J.; van Kan, F. J. P. M.; Platner, G. R.; Pinto, J. D. BioControl 1999, 43, 421-440. doi:10.1023/A:1009937108715

5. Rohi, L.; Pintureau, B. J. Appl. Entomol. 2003, 127, 265-268. doi:10.1046/j.1439-0418.2003.00753.x

6. Milonas, P. G.; Martinou, A. F.; Kontodimas, D. C.; Karamaouna, F.; Konstantopoulou, M. A. Ann. Entomol. Soc. Am. 2009, 102, 1145-1150. doi:10.1603/008.102.0624

7. Kainoh, Y. In Pheromones of non-leptidopteran insects associated with agricultural plants; Hardie, J.; Minks, A., Eds.; CABI Publishing: Wallingford, UK, 1999; pp 383-404.

8. Ruther, J. In Chemical ecology of insect parasitoids; Wajnberg, E.; Colazza, S., Eds.; John Wiley \& Sons: Chichester, UK, 2013; pp 112-144.

9. Ruther, J.; Thal, K.; Steiner, S. J. Chem. Ecol. 2011, 37, 161-165. doi:10.1007/s10886-010-9898-8

10. Kühbandner, S.; Sperling, S.; Mori, K.; Ruther, J. J. Exp. Biol. 2012, 215, 2471-2478. doi:10.1242/jeb.071217

11. Keeling, C. I.; Plettner, E.; Slessor, K. N. Top. Curr. Chem. 2004, 239, 133-177. doi:10.1007/b95452

12. van Beek, T. A.; Silva, I. M. M. S.; Posthumus, M. A.; Melo, R. J. Chromatogr., A 2005, 1067, 311-321. doi:10.1016/j.chroma.2004.09.030

13. Ukaji, Y.; Narasaka, K. Bull. Chem. Soc. Jpn. 1988, 61, 571-573. doi:10.1246/bcsj.61.571

14. Fouquet, G.; Schlosser, M. Angew. Chem., Int. Ed. Engl. 1974, 13, 82-83. doi:10.1002/anie.197400821

15. Haag, G.; Luu, B.; Hetru, C. J. Chem. Soc., Perkin Trans. 11988 , 2353-2363. doi:10.1039/p19880002353

16. Francke, W.; Schulz, S. Pheromones of Terrestrial Invertebrates. In Comprehensive Natural Products II - Chemistry and Biology; Mander, L.; Lui, H.-W.; Mori, K., Eds.; Elsevier: Oxford, 2010; Vol. 4, pp 153-223. doi:10.1016/B978-008045382-8.00095-2

17. Bartelt, R. J.; Dowd, P. F.; Plattner, R. D.; Weisleder, D. J. Chem. Ecol. 1990, 16, 1015-1039. doi:10.1007/BF01021008

18. Morris, B. D.; Smyth, R. R.; Foster, S. P.; Hoffmann, M. P.; Roelofs, W. L.; Franke, S.; Francke, W. J. Nat. Prod. 2005, 68, 26-30. doi:10.1021/np049751v

19. Mori, K.; Kuwahara, S. Tetrahedron 1986, 42, 5545-5550. doi:10.1016/S0040-4020(01)88158-8

20. Titze, J. Identifizierung und Synthese von Signalstoffen aus Insekten. Ph.D. Thesis, Universität Hamburg, Hamburg, Germany, 2007.

21. Chow, S.; Fletcher, M. T.; Lambert, L. K.; Gallagher, O. P.; Moore, C. J.; Cribb, B. W.; Allsopp, P. G.; Kitching, W. J. Org. Chem. 2005, 70, 1808-1827. doi:10.1021/jo0481093

22. Schmidt, Y.; Lehr, K.; Colas, L.; Breit, B. Chem.-Eur. J. 2012, 18, 7071-7081. doi:10.1002/chem.201103988

23. Markiewicz, J. T.; Schauer, D. J.; Löfstedt, J.; Corden, S. J.; Wiest, O.; Helquist, P. J. Org. Chem. 2010, 75, 2061-2064. doi:10.1021/jo902422y

24. Francke, W.; Dettner, K. Top. Curr. Chem. 2005, 240, 85-166. doi:10.1007/b98316

25. Akibo, A.; Masamune, S. Tetrahedron Lett. 1996, 37, 1081-1084. doi:10.1016/0040-4039(95)02353-4

26. Magnin-Lachaux, M.; Tan, Z.; Liang, B.; Negishi, E. Org. Lett. 2004, 6, 1425-1427. doi:10.1021/ol0497483
27. Lum, T.-K.; Wang, S.-Y.; Loh, T.-P. Org. Lett. 2008, 10, 761-764. doi:10.1021/ol702715q

28. Weise, C. F.; Pischl, M. C.; Pfaltz, A.; Schneider, C. J. Org. Chem. 2012, 77, 1477-1488. doi:10.1021/jo202330b

29. Schulz, C. M.; Lehmann, L.; Blatrix, R.; Jaisson, P.; Hefetz, A.; Francke, W. J. Chem. Ecol. 2002, 28, 2541-2555. doi:10.1023/A:1021492204400

30. Geerdink, D.; Buter, J.; van Beek, T.; Minnaard, A. J. Beilstein J. Org. Chem. 2014, 10, 761-766. doi:10.3762/bjoc.10.71

31. Pompanon, F.; De Schepper, B.; Mourer, Y.; Fouillet, P.; Bouletreau, M. J. Chem. Ecol. 1997, 23, 1349-1360. doi:10.1023/B:JOEC.0000006468.19993.70

\section{License and Terms}

This is an Open Access article under the terms of the Creative Commons Attribution License (http://creativecommons.org/licenses/by/2.0), which permits unrestricted use, distribution, and reproduction in any medium, provided the original work is properly cited.

The license is subject to the Beilstein Journal of Organic Chemistry terms and conditions: (http://www.beilstein-journals.org/bjoc)

The definitive version of this article is the electronic one which can be found at: doi:10.3762/bjoc. 10.72 